\title{
Novel method for state selective determination of electron-impact-excitation cross sections from $0^{\circ}$ to $180^{\circ}$
}

\author{
Marvin Weyland ${ }^{1,2^{*}}$, Xueguang Ren ${ }^{1,2}$, Thomas Pflüger ${ }^{1,2}$, Woon Yong Baek ${ }^{1}$, Klaus Bartschat ${ }^{3}$, \\ Oleg Zatsarinny ${ }^{3}$, Dmitry V Fursa ${ }^{4}$, Igor Bray ${ }^{4}$, Hans Rabus ${ }^{1}$ and Alexander Dorn ${ }^{2}$
}

\author{
*Correspondence: \\ weyland@mpi-hd.mpg.de \\ 1 Physikalisch-Technische \\ Bundesanstalt, Bundesallee 100, \\ 38116 Braunschweig, Germany \\ ${ }^{2}$ Max-Planck-Institut für Kernphysik, \\ Saupfercheckweg 1, 69117 \\ Heidelberg, Germany \\ Full list of author information is \\ available at the end of the article
}

\begin{abstract}
We use an improved target recoil momentum spectroscopy setup to determine differential cross sections for excited metastable state production in atoms and molecules by electron impact and show its capabilities for an atomic helium target. A crossed beam setup with a supersonic helium jet and a pulsed electron beam at energies close to the excitation threshold of $19.82 \mathrm{eV}$ was used. Measuring the recoil momentum vector of the target instead of the momentum of the scattered electron removes common restrictions to the accessible scattering angles while the microchannel plate detector ensures a high counting efficiency. Using a photoemission electron source we reach an energy resolution of about $200 \mathrm{meV}$ at $1 \mu \mathrm{A}$ peak current. Results are compared with simulations using theoretical convergent-close-coupling (CCC), R-matrix with pseudo-states (RMPS) and B-spline R-matrix (BSR) calculations and show good agreement.
\end{abstract}

Keywords: Inelastic scattering; Electron impact excitation

\section{Background}

In traditional electron impact experiments, the scattered electron is measured using a movable detector. The scattering angles are scanned by changing the position of the detector [1]. This technique allows measurement within a limited angular range, as the electron detector would not be able to measure the scattered electrons in the backward and forward directions due to the interference of the spectrometer with the incoming or outgoing projectile beam. Detecting the recoil momentum of the excited target instead of the scattered electron has the advantage that the projectile beam has no influence on the measurement. This principle has been shown by Murray and Hammond [2,3] for electron impact excitation at intermediate projectile energies. Our improved setup allows for state selective measurements close to the threshold energy by using a time- and positionsensitive microchannel plate detector, where events from all electron scattering angles can be registered simultaneously and by using a photo-emission electron source.

Electron impact excitation is the simplest inelastic scattering process to study experimentally, as it has only two free particles before and after the collision. To satisfy momentum conservation, the projectile can only transfer its momentum to the target.

(c) 2014 Weyland et al.; licensee Springer. This is an Open Access article distributed under the terms of the Creative Commons Attribution License (http://creativecommons.org/licenses/by/2.0), which permits unrestricted use, distribution, and reproduction in any medium, provided the original work is properly cited. 
Because of the large mass of the target compared to the mass of the projectile electron, the corresponding transfer of kinetic energy to the target is negligible. The electron with the initial energy $E_{0}^{e}$ loses the energy $E_{\text {exc }}$ needed to excite a certain state in the target and remains with the energy $E_{1}^{e}$ after the collision:

$$
E_{1}^{e}=E_{0}^{e}-E_{\mathrm{exc}} \text {. }
$$

The momentum $\mathbf{q}$, which is transferred to the target in the process, is

$$
\mathbf{q}=\mathbf{p}_{0}^{e}-\mathbf{p}_{1}^{e},
$$

where $\mathbf{p}_{0}^{e}$ and $\mathbf{p}_{1}^{e}$ are the momenta of the projectile electron before and after the collision, respectively. The absolute values of these momenta depend only on the corresponding electron energies:

$$
\begin{aligned}
& \left|\mathbf{p}_{0}^{e}\right|=\sqrt{2 m_{e} E_{0}^{e}} \\
& \left|\mathbf{p}_{1}^{e}\right|=\sqrt{2 m_{e}\left(E_{0}^{e}-E_{\text {exc }}\right)},
\end{aligned}
$$

where $m_{e}$ is the electron mass. All relevant momenta are depicted in Figure 1, with the incoming electron beam moving along the positive $y$-direction and the target gas jet moving along the positive z-direction. Scattered excited atoms in metastable states are detected by the microchannel plate (MCP) detector indicated as the light gray disc. Before the collision, the target has the initial momentum $\mathbf{p}_{0}^{t}$ in the gas jet. As a result of the momentum transfer in the collision, its final momentum is

$$
\mathbf{p}_{1}^{t}=\mathbf{p}_{0}^{t}+\mathbf{q}=\mathbf{p}_{0}^{t}+\mathbf{p}_{0}^{e}-\mathbf{p}_{1}^{e} .
$$

For a given projectile momentum $\mathbf{p}_{0}^{e}$ and a particular excited state energy $E_{\mathrm{exc}}$, the absolute value of the final electron momentum $\left|\mathbf{p}_{1}^{e}\right|$ is fixed. The target momenta therefore lie on a sphere with origin $\left(\mathbf{p}_{0}^{t}+\mathbf{p}_{0}^{e}\right)$ and radius $\left|\mathbf{p}_{1}^{e}\right|$ for all possible scattering angles. In Figure 1, momentum transfer is shown only in the $x-y$ plane for simplicity: therefore, all events that correspond to the excitation of the same state have target momenta on the dotted dark gray circle while the momentum of the outgoing electron lies on the full circle. The radius of this circle is proportional to the amount of electron momentum after the collision, putting energetically lower lying states - which correspond to a higher excess energy - on larger radii.

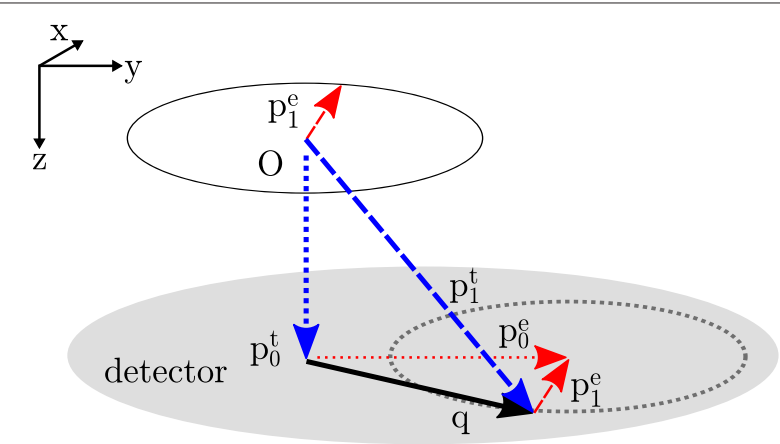

Figure 1 Overview of all momenta of electrons and targets before and after the collision. Collision center is at $\mathrm{O}$. For simplicity, only momentum transfer in the $\mathrm{x}-\mathrm{y}$ plane is shown. Initial momenta are shown in dotted lines, final momenta in dashed lines, and the momentum transfer is shown in a solid line. Target momenta are shown in blue (thick), projectile momenta in red (thin). 
Momentum in $\mathrm{x}$ - and $\mathrm{y}$-direction can be calculated from the impact position of the excited target on the detector and its time-of-flight $t_{f}$ :

$$
q_{x}=m_{t} \frac{x}{t_{f}} \quad q_{y}=m_{t} \frac{y}{t_{f}} .
$$

For these directions perpendicular to the gas jet, a high momentum resolution of 0.068 a.u. ${ }^{\text {a }}$ can be reached. Momentum transfer in z-direction can be calculated by

$$
\mathrm{q}_{z}=p_{0, z}^{t}-\frac{m_{t} d}{t_{f}}
$$

where $d$ is the distance between the interaction region and the detector plane. Unfortunately, our measurements showed that the initial longitudinal momentum spread in the supersonic jet due to its finite temperature is about 0.75 a.u. and therefore of the same order of magnitude as the transferred momentum, making the momentum transfer in $\mathrm{z}$-direction inaccessible for all practical purposes. The rotational symmetry of the electron scattering cross section around the y-axis, however, allows for reconstruction of the complete differential cross section, as will be described in Section 'Data analysis'.

Helium has long been a benchmark target for the investigation of electron-atom collisions due to its simple structure. Many experiments have been conducted to measure electron impact excitation cross sections [2,4-10] and theories were developed to explain the experiments [11-13]. Total cross sections are known very precisely from experiment [8] and agree very well with theoretical calculations [12], but measurement of differential scattering cross sections has for a long time been restricted to an intermediate range of projectile scattering angles, usually ranging from $10^{\circ}$ to $130^{\circ}[7,10]$.

One way of accessing electron scattering angles around $180^{\circ}$ is the use of a magnetic angle changer [14], in which a strong localized magnetic field in the interaction region changes the ejection direction of the electrons, depending on their energy. The few experiments that have been conducted to measure inelastic electron scattering on helium using this technique [15-17] show discrepancies between experiment and theory especially at high scattering angles. Detection of the scattered helium atoms, as performed with the instrument described here, is therefore a sensible addition to the existing methods.

In earlier experiments, the momentum transfer to the scattered helium was used to determine electron scattering angles by Zajonc et al. [9]. They only measured the timeof-flight of metastable helium atoms, which varied due to the momentum transfer in the flight direction of the helium. This method gave access to all scattering angles, but was unable to distinguish between different excited states and was therefore only applicable in the energy range from $19.8 \mathrm{eV}$ to $20.6 \mathrm{eV}$, where just the $2^{3} \mathrm{~S}$ state can be excited. Murray and Hammond [18] used a rotatable detector setup to measure the deflection angle of helium atoms after electron impact excitation. In their arrangement the incoming electron beam crosses a gas jet perpendicularly and electron scattering within the plane determined by both beams is studied. Thus, scattered electron momentum determination relies on the measurement of the atomic recoil momentum along the incoming electron beam and along the gas jet. Since the resolution along the latter direction, due to the thermal velocity spread, is limited to about 0.76 a.u., a larger projectile excess energy was chosen such that different electron scattering angles result in more strongly varying recoil momenta. Also, excitation to the different accessible states was not resolved but summed differential cross sections were obtained. 
Using a microchannel-plate detector with time of flight resolution and $x-y$-position resolution for detection of the recoiling metastable excited atoms, we obtain $4 \pi$-acceptance for electron scattering. In the plane perpendicular to the gas jet we obtain strongly improved momentum resolution, discriminating excitation of different states at low energies above the excitation threshold.

Our new setup is not limited to a helium target, but rather it can be used with all light targets, for which the deflection from their original direction after the collision is large enough to be resolved.

\section{Results and discussion}

The recorded momenta associated with the excitation of metastable states provide diverse information. On the one hand, the excited atom yield and, thus, the total metastable state excitation cross section as a function of impact energy are obtained. On the other hand, excited state resolved cross sections can be extracted as, e.g., excitation functions and cross sections differential in the projectile scattering angle.

In Figure 2, the total yield of excited helium atoms is depicted as a function of projectile energy. The metastable state production starts at the excitation threshold of the lowest $2^{3} \mathrm{~S}$ state and shows a characteristic shape due to negative ion resonances and additional excited states whose energies are marked in the diagram. The features of this curve are well known and described, e.g., by Brunt et al. [8]. In the energy range shown, only the $2^{1} \mathrm{P}$ state at $21.2 \mathrm{eV}$ decays to the ground state before detection and is therefore ignored. The red solid line in the diagram represents the theoretical cross section obtained from an RMPS calculation after convolution with a Gaussian describing the energy spread of the projectile beam. Expected helium yield curves at higher and lower energy resolution are shown for comparison as well. Energy spread and an energy offset were obtained by fitting the convolved theoretical curve to the experimental data. The FWHM of the energy spread varied according to the operating conditions of the electron gun between $150 \mathrm{meV}$ and $250 \mathrm{meV}$, and is $200 \mathrm{meV}$ in Figure 2. The energy offset arises due to contact potentials in the cathode and in the interaction region.

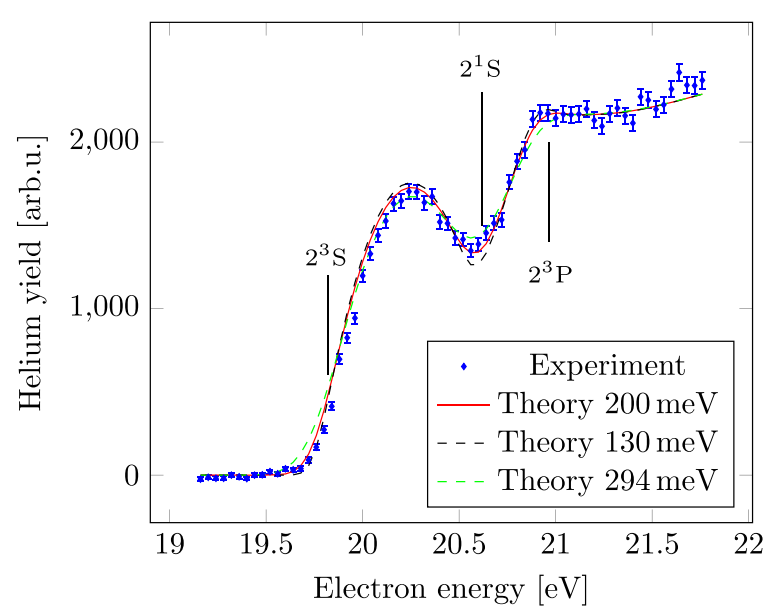

Figure 2 Total electron impact excitation cross section. Comparison of measured total yield of excited helium atoms with theoretical total excitation cross sections after the convolution with the impact energy spread of $200 \mathrm{meV}$ (FWHM). This comparison is used to determine the energy spread of the electron beam. 
Figure 3(a) shows the experimental cross section as a function of scattered electron momentum $\mathbf{p}_{1}^{e}$ for an impact energy of $E_{0}=22.0 \mathrm{eV}$. It shows the projection of all momenta onto the detector plane. In Figure 3(b), the out-of-plane scattering contribution was restricted by including only events close to the velocity distribution maximum as described in Section 'Data analysis'. The various features that correspond to different excited states can be separated better than in the projection of all events. Theoretical cross sections displayed in Figure 3(c) and 3(d) are CCC and RMPS calculations, respectively. In these plots, scattered electron momenta for the excitation of a particular atomic state lie on a circle with a certain radius matching the corresponding excess energy. Circles for the three accessible states $2^{3} \mathrm{~S}$ (solid line), $2^{1} \mathrm{~S}$ (dashed line) and $2^{3} \mathrm{P}$ (dotted line) are shown in the plots. Scattering leading to excitation of the $2^{3} \mathrm{~S}$ state can be separated from the higher lying $2^{1} \mathrm{~S}$ and $2^{3} \mathrm{P}$ states. These, on the other hand, are fairly closely spaced with energies of $20.616 \mathrm{eV}\left(\left|\mathbf{p}_{1}^{e}\right|=0.319\right.$ a.u. $)$ and $20.964 \mathrm{eV}\left(\left|\mathbf{p}_{1}^{e}\right|=0.276\right.$ a.u. $)$ and cannot be resolved due to the combined uncertainties of impact energy and target size. The cross section for the excitation of the $2^{3} \mathrm{~S}$ state exhibits maxima at roughly $\pm 90^{\circ}$ scattering angle and for backward scattering. The excitation cross sections for $2^{1} \mathrm{~S}$ and $2^{3} \mathrm{P}$ states show a strong and relatively sharp maximum in the forward direction, while the maximum in the backward direction extends over a larger angular range. Experimental results are in excellent agreement with the theories.

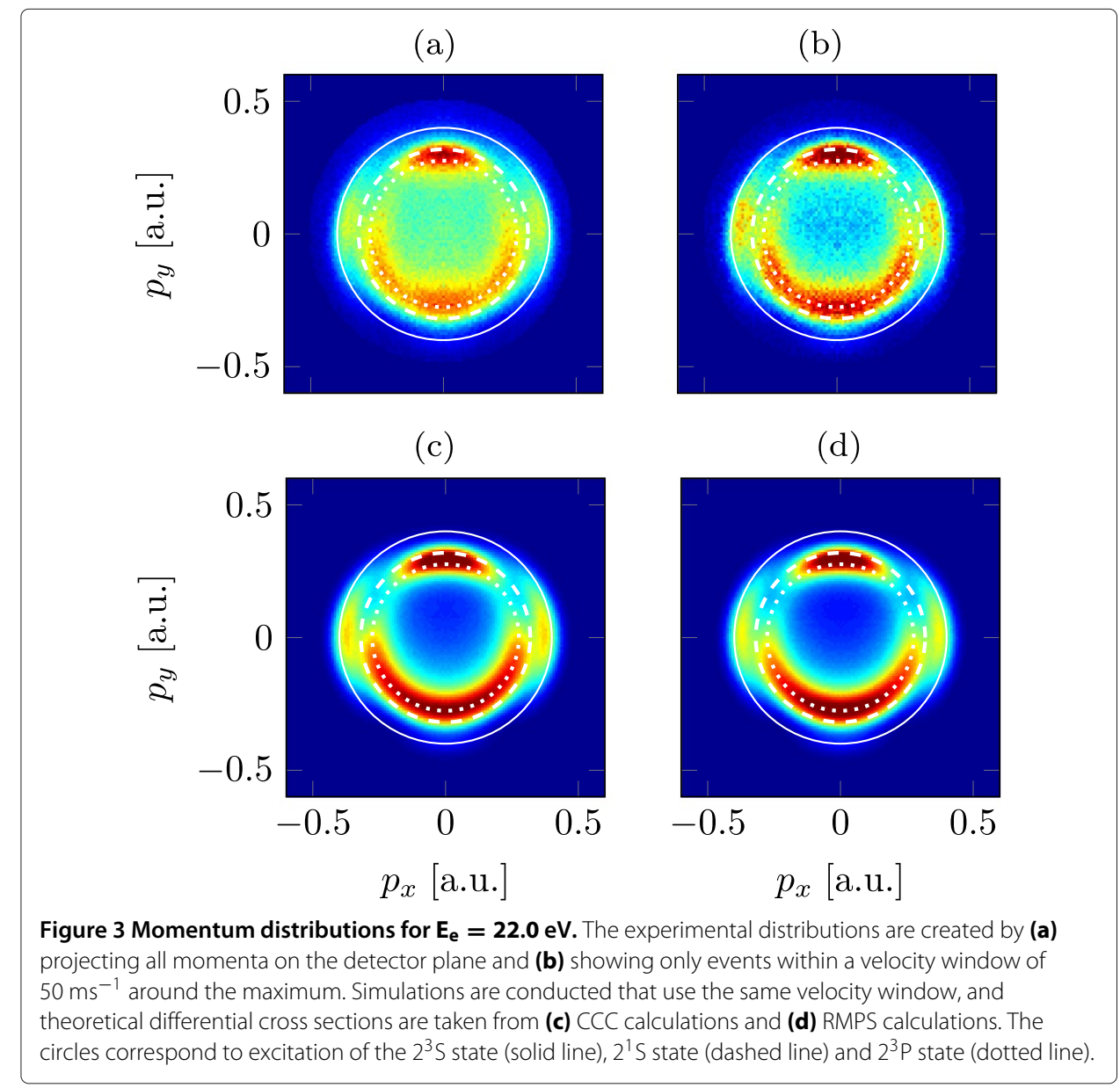


In Figure 4, the differential cross sections for the excitation of $2^{1} \mathrm{~S}$ and $2^{3} \mathrm{P}$ states are shown. The data were obtained by integrating the yield of metastable excited helium atoms over the energy range $(1.2 \pm 0.05) \mathrm{eV}$ of the scattered electrons for both the experimental data and the theoretical CCC, RMPS and BSR data. The total cross section was not determined. Therefore, theoretical and experimental data sets were normalized to yield the same integrated cross section. Figure 5 depicts the data for $(2.2 \pm 0.05) \mathrm{eV}$ excess energy, obtained in the same way and corresponding to the excitation of $2^{3} \mathrm{~S}$ state.

There is no significant difference between CCC, RMPS and BSR calculations in the differential cross section for $2^{3} \mathrm{~S}$ state excitation, and the experiments are in good agreement with all theories. For the $2^{1} \mathrm{~S}$ and $2^{3} \mathrm{P}$ state excitation, however, the $\mathrm{CCC}$ calculations show lower yield in the broad backward scattering peak and a more pronounced peak at about $110^{\circ}$, showing a reminiscent feature of the $2^{3} \mathrm{~S}$ state excitation (cf. Figure 4). However, all theories are in good agreement with the experimental results within our measurement uncertainty.

Finally, Figure 6 displays results from the data analysis using the inverse Abel transformation. In Figure 3(a), the experimental data are shown for the projection of all recorded momenta onto the detector plane ( $x$-y plane). Applying the inverse Abel transformation to this data, the pure $\mathrm{x}-\mathrm{y}$ plane cross section $\sigma\left(p_{x}, p_{y} ; p_{z}=0\right)$ displayed in Figure 6(a) is obtained. A simulation of the experiment using the RMPS calculation is shown in Figure 6(b). It is evident from the comparison with Figure 3 that the inverse Abel transformation provides a better resolution of the different states.

\section{Conclusions}

Our setup allows for the measurement of electron impact excitation to metastable states in light targets, resolving all scattering angles as well as different excited states. We demonstrated the setup's ability by using a helium target. Differential cross sections for the excitation of the $2^{1} \mathrm{~S}$ and $2^{3} \mathrm{P}$ state in helium close to the excitation threshold energy are shown for the first time at all scattering angles, including the scattering angles in the

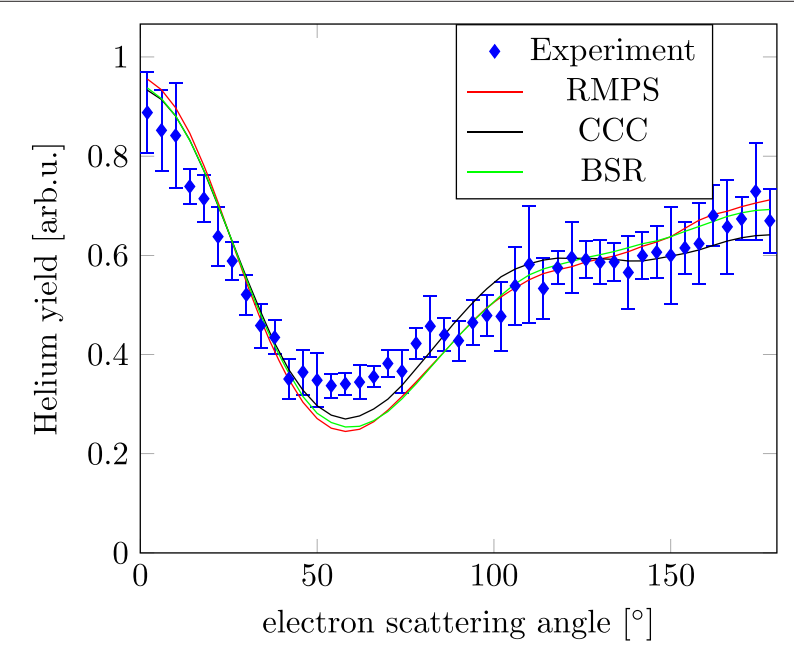

Figure 4 Differential excitation cross sections for an excess energy of $\mathbf{1 . 2} \mathbf{~ e V}$. The graphs shown are: present experiment (blue points), RMPS calculation (red line), CCC calculation (black line), BSR calculations (green line). The three data sets were normalized such that they yield the same total excitation cross section. 


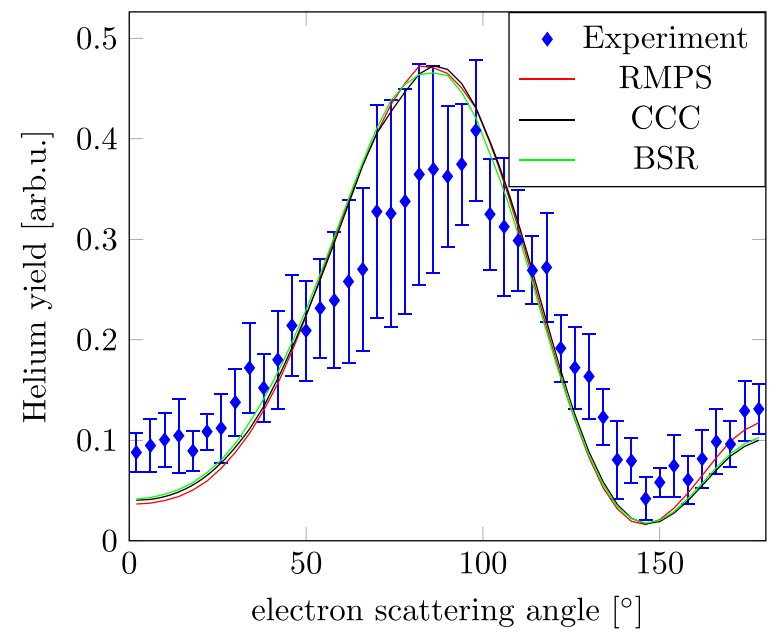

Figure 5 Differential excitation cross sections for an excess energy of $2.2 \mathrm{eV}$. Same as Figure 4 , but with $2.2 \mathrm{eV}$ excess energy corresponding to excitation of the $2^{3} \mathrm{~S}$ state.

vicinity of $0^{\circ}$ and $180^{\circ}$. This instrument provides an additional tool to check theoretical predictions of electron impact excitation cross sections.

\section{Methods}

\section{Setup}

The experimental setup (cf. Figure 7) consists of an electron beam from a pulsed photoemission electron source, crossed with a continuous supersonic gas jet, a potentially variable interaction region and a time and position-sensitive MCP detector.

We have created a supersonic jet of the target gas by expansion through a $30 \mu \mathrm{m} \mathrm{noz-}$ zle at a backing pressure of 5 bar. The jet is collimated by two skimmers $250 \mu \mathrm{m}$ and $400 \mu \mathrm{m}$ in diameter. Each skimmer is set in a differentially pumped stage, maintaining pressures of $2 \times 10^{-3} \mathrm{mbar}$ in the first jet stage, $5 \times 10^{-6} \mathrm{mbar}$ in the second jet stage, and $5 \times 10^{-8} \mathrm{mbar}$ in the main chamber during operation. The longitudinal jet temperature

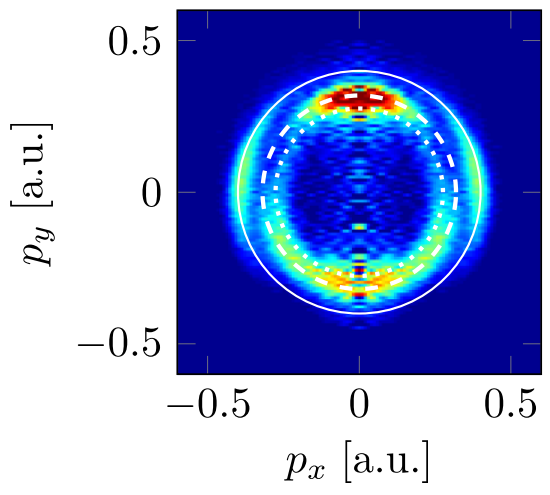

(a)

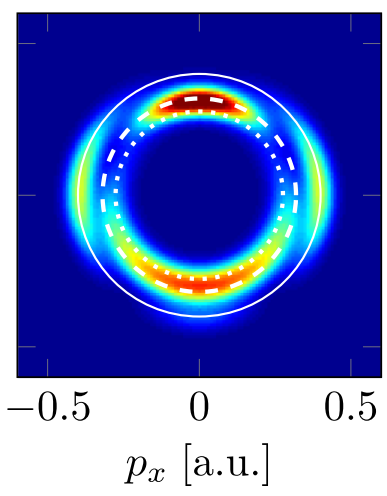

(b)

Figure 6 Reconstructed electron momentum distributions using the inverse Abel transform. Electron momentum distributions are shown for an impact energy of $E_{e}=22.0 \mathrm{eV}$ (a) after reconstructing the distribution in the plane using the inverse Abel transform. (b) Simulation of the experiment using RMPS calculations when scattering only in the detector plane. 


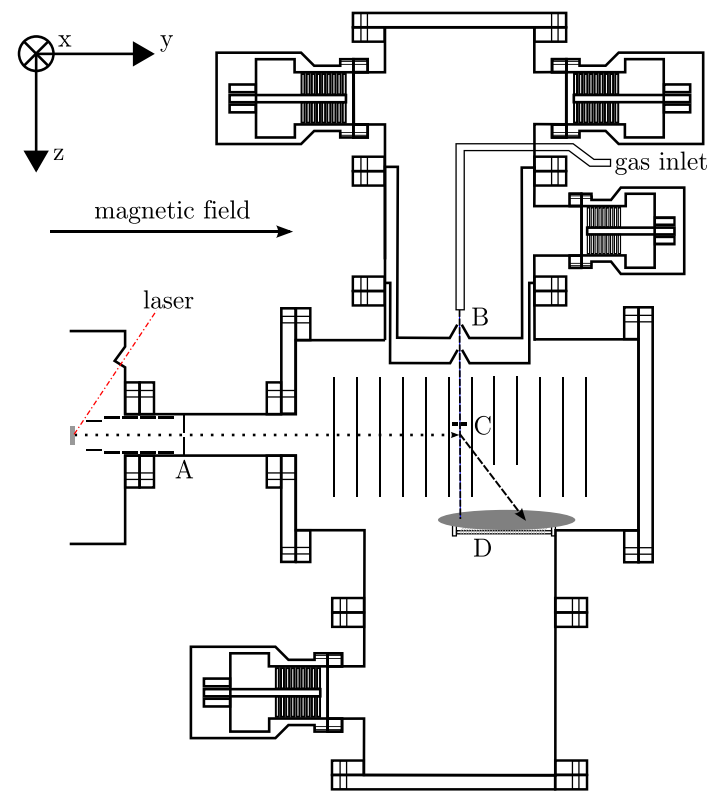

Figure 7 Schematic view of the setup. Electrons (dotted lines) are created in the photoemission gun in a separate vacuum chamber (left), focused on the aperture (a) and guided to the interaction region by a magnetic field (solid line). The supersonic helium jet (dashed line) is created by two skimmers after a nozzle (b) and crosses the electron beam inside the variable potential interaction region (c), where it is additionally collimated by a third aperture. Excited target atoms are detected by an MCP with delay line anodes at the bottom (d), allowing precise measurement of the deflection after the electron impact.

is $3 \mathrm{~K}$. With this setup a beam diameter of about $2 \mathrm{~mm}$ and a target number density of $1.5 \times 10^{11} \mathrm{~cm}^{-3}$ can be obtained in the region of interaction with the electron beam. To increase resolution, the beam is additionally collimated by inserting a $500 \mu \mathrm{m}$ aperture a few millimeters above the interaction point, which is held at the same potential as the surrounding region at all times. The beam is dumped directly into the chamber without an additional dump stage.

The electron beam is produced in a photoemission electron gun [19]. A GaAs cathode is coated with a monolayer of cesium and oxygen to obtain negative electron affinity (NEA) conditions. In a NEA semiconductor, the conduction band minimum is above vacuum energy. Illuminating the surface with a laser excites electrons to the conduction band and can induce electron emission by tunneling through the thin surface barrier. The electron gun has been described in detail in the work by Schröter et al. [20]. To keep the surface stable, ultra-high vacuum is needed. The electron gun is therefore placed in a separately pumped chamber, which can be sealed off completely from the measurement vacuum chamber by a gate valve. This so-called gun chamber was heated to $220^{\circ} \mathrm{C}$ for one week to reach a base pressure below $1 \times 10^{-11}$ mbar at room temperature afterwards. During the measurements, the two chambers are connected by an aperture $1 \mathrm{~mm}$ in diameter, which allows a pressure of $1 \times 10^{-10} \mathrm{mbar}$ to be maintained in the electron gun chamber.

The pulsed electron beam is produced by a laser pulse, illuminating the cathode. Previous studies have shown that multi-mode lasers can induce a high energy spread in the electron beam, as do high current densities [21]. Kolac et al. [22] explain this large energy spread from multi-mode lasers with high temporal intensity fluctuations which lead to an inhomogeneous space charge distribution in the emission current. 
In our setup, the cathode is illuminated by a single-longitudinal-mode laser with a constant temporal intensity profile. The laser has a wavelength of $671 \mathrm{~nm}$ corresponding to a photon energy of $1.84 \mathrm{eV}$, which is high enough to populate the conduction band in the used crystal that has a band gap of $1.42 \mathrm{eV}$ [23]. When operated in continuous-wave mode at a laser intensity of $25 \mathrm{~mW}$, currents of several $\mu \mathrm{A}$ are reached. The pulsed electron beam is produced by guiding the incident laser through an acousto-optic modulator and coupling the light of the first diffraction maximum into an optic fiber, where it is guided to the focussing optics outside the vacuum chamber. Using the acousto-optic modulator allows switching the laser within $50 \mathrm{~ns}$ and obtaining extinction ratios better than $10^{-3}$. The laser is focused to a diameter of $1 \mathrm{~mm}$ at the cathode, which restrains the electron source to an area of $1.4 \mathrm{~mm} \times 1.0 \mathrm{~mm}$, due to the laser incidence angle of $45^{\circ}$. Additional attenuation filters and an aperture are used to regulate the laser beam intensity such as to compensate for a decreasing quantum efficiency of the cathode over time.

The electrons are focused by four electrostatic lenses and two pairs of deflection plates onto the $1 \mathrm{~mm}$ aperture, which separates the electron gun chamber from the detection chamber. Additional steering to the interaction point is supplied by a pair of Helmholtz coils, creating a magnetic field of $1.2 \mathrm{mT}$ pointing from the center of the aperture to the center of the interaction region, thereby confining the electron beam motion perpendicular to the direction of the magnetic field. Fine control of the beam position in $\mathrm{x}$ - and $\mathrm{z}$-direction is possible with two additional pairs of coils that create a low magnetic field up to $0.1 \mathrm{mT}$ perpendicular to the direction of the electrons.

The excitation process takes place in an electric-field-free region that is provided by a number of electrically connected metal rings. The distance between two adjacent metal rings is $10 \mathrm{~mm}$ and the symmetry axis of the rings is coincident with the electron beam direction. The rings are set to a variable voltage to change the electron impact energy. The electric-field-free region between the two central rings is accessible to the electron beam and the target jet. Rings blocking the flight path of the scattered target atoms are cut on the lower end to clear the path to the detector. The electron gun, interaction region boundary rings and the magnetic field are aligned precisely to keep the projectile beam on the axis of the apparatus when the interaction potential is changed. Any change in overlap of the electron beam and the jet leads to changing count rates. In the aligned setup, this effect was negligible for scanning amplitudes up to $2 \mathrm{~V}$. The scanning voltage is read by an analog-to-digital converter (Hytec ADC 521).

After the inelastic electron-atom scattering, the excited atoms impinge on an MCP detector $80 \mathrm{~mm}$ in diameter with a position-sensitive delay line anode, located about $110 \mathrm{~mm}$ below the interaction region. Here they are efficiently detected since their internal energy gives rise to electron release at the detector surface. It is therefore necessary to shield the MCP from other electrons. An additional grid in front of the detector, set to $-200 \mathrm{~V}$, and the presence of the magnetic field eliminate background due to charged particles. The $\mathrm{MCP}$ is mounted at $32 \mathrm{~mm}$ offset of the target jet in positive y-direction to account for momentum transfer to the atoms and to detect excited atoms for all projectile scattering angles at excess energies up to $30 \mathrm{eV}$. Time-of-flight information and delay line signals are read by a time-to-digital converter (LeCroy 3377 TDC) operated in common stop mode and gated to accept only events in the expected time-of-flight range from $55 \mu \mathrm{s}$ to $87 \mu \mathrm{s}$. Data from the TDC and ADC are read into a personal computer by a CAMAC system and are analyzed in offline mode. To correct for varying detection efficiency at 
different positions of the delay line anodes, the detector was homogeneously illuminated using an ${ }^{241} \mathrm{Am} \alpha$-source. The efficiency map obtained this way was used to weigh counts in the electron-scattering measurements.

\section{Experiments on helium}

Measurements were conducted at an $11 \mathrm{kHz}$ gun pulse repetition rate and with electron pulse lengths ranging from $50 \mathrm{~ns}$ to $100 \mathrm{~ns}$, which are needed to precisely measure the time-of-flight of the excited atoms. Time-of-flight and the position on the MCP detector are then used to calculate the projection of the momentum transfer $\mathbf{q}$ on the detector plane. The pulse length is also considerably shorter than the width of the time-of-flight distribution of the excited helium atoms that originate from the thermal velocity spread in the jet and the momentum transfer along the jet direction. The initial velocity distribution of the jet is therefore assumed to be equal to the measured velocity distribution at the excitation threshold. As can be seen from Eq. (4), the complete momentum of the electron is transferred to the helium, if $E_{0}^{e}=E_{\text {exc }}$. In this case, no momentum spread arises due to different scattering angles. The velocity distribution for this case is shown in Figure 8 . The mean jet velocity is $1624 \mathrm{~ms}^{-1}$ and the distribution has a full width at half maximum of $220 \mathrm{~ms}^{-1}$, corresponding to a longitudinal jet temperature of $3.0 \mathrm{~K}[24,25]$. In the simulation discussed in the next section, these jet characteristics are used as one source of the momentum spread.

The cathode potential of the electron gun is fixed to $-22.9 \mathrm{~V}$. Different impact energies are created by changing the potential of the interaction region while keeping all electron gun settings. For impact energy scans, a triangular scan with a $1 \mathrm{~Hz}$ repetition rate is carried out on the voltage applied to the interaction region boundary, usually providing the energy scan range from $19 \mathrm{eV}$ to $23 \mathrm{eV}$. Energy scans are used to measure the electronimpact-energy dependence of the total cross section for metastable state excitation that is used to calibrate the impact energy scale based on RMPS calculations. From the measured energy dependence of the cross section the width of the electron energy distribution is

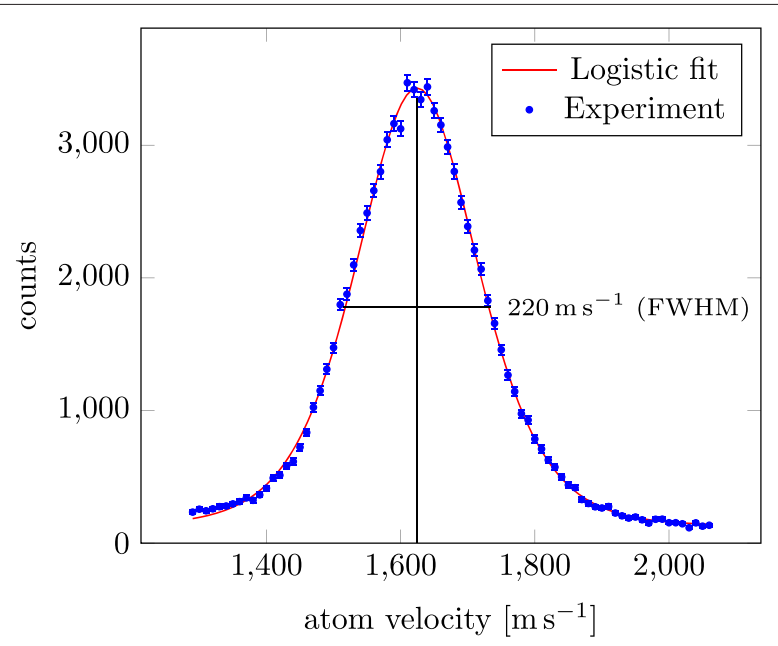

Figure $\mathbf{8}$ Velocity distribution in the target jet. The velocity distribution is determined from the time-of-flight distribution of helium excited by electrons with threshold energy, and the logistic fit to the experimental data is used in simulations of the experiment. The mean velocity is $1624 \mathrm{~ms}^{-1}$ and the full width at half maximum (FWHM) of the distribution is $220 \mathrm{~ms}^{-1}$. 
determined by comparison with theoretical cross-sectional data [12] that are convoluted with Gaussian distributions of different standard deviation.

Differential cross sections were measured with constant impact energy and count rates of around 300 counts per second were achieved. The counts were accumulated over an acquisition period of 10 to 24 hours. The discussed exemplary cross sections were obtained for an impact energy $E_{0}^{e}$ of $22.0 \mathrm{eV}$. At this energy, theoretical calculations predict only minor changes in differential scattering cross sections within several $100 \mathrm{meV}$, making the measurement less sensitive to the finite energy spread of the electron beam.

Flight times in this setup are about $70 \mu \mathrm{s}$, making the measurement insensitive to fast decaying states like the $2^{1} \mathrm{P}$ state in helium, which has a radiative lifetime of $0.6 \mathrm{~ns}$ [26] and decays to the undetected ground state. The three lowest excited states $2^{3} \mathrm{~S}, 2^{1} \mathrm{~S}$ and $2^{3} \mathrm{P}$ are metastable because radiative dipole transitions are forbidden. They have sufficiently long lifetimes of about $8000 \mathrm{~s}\left(2^{3} \mathrm{~S}\right)$ and $20 \mathrm{~ms}\left(2^{1} \mathrm{~S}\right)$ and are detected before a significant amount of the excited metastable atoms decays $[27,28]$. The $2^{3} \mathrm{P}$ state radiatively decays with a lifetime of about $100 \mathrm{~ns}[8,26]$ to the $2^{3} \mathrm{~S}$ state, which is then detected.

The $2^{1} \mathrm{P}$ state produces photons of $21.2 \mathrm{eV}$ when it decays. These photons can be detected by the $\mathrm{MCP}$, but are not recognized, because they arrive before the start of the TDC gate and are therefore ignored.

\section{Data analysis}

In the experiment, the momentum transfer in the detector plane is measured very accurately. Error propagation analysis for Eq. (6) with typical values results in an uncertainty of $\Delta q_{x, y}=0.068$ a.u. that originates mainly from the transversal size of the gas jet. Analogously, the uncertainty of the momentum in the jet direction given by Eq. (7) is an order of magnitude higher: $\Delta q_{z}=0.8$ a.u., which is almost exclusively due to the initial velocity distribution in the gas jet. Because the highest transferred momentum is 0.4 a.u. at $2.2 \mathrm{eV}$ excess energy, no useful information about the momentum transfer in z-direction is available.

Therefore, in the analysis, projections of the momenta on the $x-y$ plane are used. Two methods of data analysis are applied. First, restricting analysis to events near the peak of the time-of-flight distribution is used to suppress large $q_{z}$-values. Second, the fact that the scattering has rotational symmetry around the $y$-axis allows the use of an inverse Abel transform $[29,30]$ to reconstruct the full 3D-scattering cross section from the $x-y$ projection, based on the axial symmetry of the scattering. Both sets of results are compared to the predictions from simulations.

The first approach offered the advantage that only little post-processing was necessary. Scattering out of the detector plane was partly suppressed by using only events with velocities in a $50 \mathrm{~ms}^{-1}$ window around the maximum of the velocity distribution (cf. Figure 8). In this case, only out-of-plane scattering events with a vertical momentum transfer up to $\left|q_{z}\right|=0.17$ a.u. contribute to the momentum transfer maps. On the other hand, atoms from the wings of the velocity distribution can contribute if they experience a vertical momentum transfer such that their resulting time-of-flight is within the acceptance interval. This contribution is smaller due to the lower number of atoms in the wings of the velocity distribution. Owing to this condition, the obtained momentum transfer maps show pronounced in-plane scattering features. 
The second way to analyze the data was to reconstruct the momentum transfer in the detector plane by exploiting the rotational symmetry around the electron impact direction. Using all events we obtained the projection of all momenta on the detector plane. The projection of a cylindrically symmetrical object on a plane that contains the symmetry axis is an Abel transformation [31]. In the experiment, the symmetry axis was along the electron impact direction in y-direction and the projection plane was the detection plane, i.e. the $x-y$ plane. The original distribution was reconstructed using the inverse Abel transform [30]. Here, the results are independent of the velocity distribution in the jet and show better momentum resolution in $\mathrm{x}$ - and $\mathrm{y}$-direction. These results are compared to a simulation where scattering was confined to the detector plane, thus eliminating the jet velocity distribution from the calculations.

To allow for a comparison of the experimental results with theoretical cross sections, the expected momentum transfer maps were simulated by a Monte Carlo-type program. This program takes into account the differential cross sections provided by R-matrix with pseudo-states (RMPS), B-spline R-matrix (BSR), and convergent-close-coupling (CCC) calculations [13], as well as the following experimental factors: target initial velocity, collision position, and collision energy for every event. Finally, the momentum of the excited target atom is mapped as a projection on the $x-y$ plane. The time-of-flight is calculated from the initial velocity of the atom and the momentum change during the collision. Events produced in this program are filtered by their time-of-flight using the same velocity condition as was used in the experiment. Thus, out-of-plane scattering is taken into account in the same way as in the experiments and the measured momentum distributions are reproduced. The finite size of the jet has been modeled as an additional momentum spread which is specific to the setup geometry. All random variables are assumed to have Gaussian or logistic distributions and parameters of the distributions are taken from measured properties where possible.

The method of using only events with a certain velocity strongly improves the visibility of details in the momentum distributions. At the same time, the result depends on experimental parameters like electron beam resolution and all jet properties. A comparison with theoretical cross sections is difficult, as all parameters enter in the simulation as well.

The momentum distributions that were reconstructed using the inverse Abel transformation were compared to a similar simulation. Scattering angles were restricted to the $\mathrm{x}-\mathrm{y}$ plane and no velocity condition was applied to reconstruct the expected momentum transfer maps for this case.

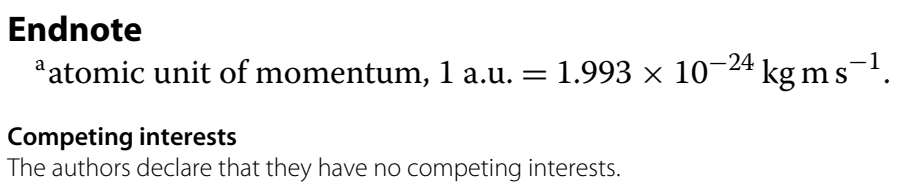


Author details

${ }^{1}$ Physikalisch-Technische Bundesanstalt, Bundesallee 100, 38116 Braunschweig, Germany. ${ }^{2}$ Max-Planck-Institut für Kernphysik, Saupfercheckweg 1, 69117 Heidelberg, Germany. ${ }^{3}$ Department of Physics and Astronomy, Drake University, Des Moines, IA 50311, USA. ${ }^{4}$ ARC Centre for Antimatter-Matter Studies, Curtin University, Perth, WA 6845, Australia.

Received: 3 April 2014 Accepted: 5 June 2014

Published: 7 August 2014

References

1. Hall Rl, Joyez G, Mazeau J, Reinhardt J, Schermann C: Electron impact differential and integral cross sections for

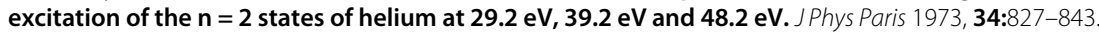

2. Murray AJ, Hammond P: Laser probing of metastable atoms and molecules deflected by electron impact. Phys Rev Lett 1999, 82:4799-4802.

3. Murray AJ, Hammond P: Studies of electron-Excited targets using recoil momentum spectroscopy with laser probing of the excited state. Adv Atom Mol Opt Phy 2001, 47:163-204.

4. Schulz GJ, Fox RE: Excitation of metastable levels in helium near threshold. Phys Rev 1957, 106:1179-1181.

5. Holt HK, Krotkov R: Excitation of $\mathbf{n}=\mathbf{2}$ states in helium by electron bombardment. Phys Rev 1966, 144(1):82-93.

6. Dugan JLG, Richards HL, Muschlitz EEJr: Excitation of the metastable states of helium by electron impact. J Chem Phys 1967, 46(1):346-351.

7. Pichou F, Huetz A, Joyez G, Landau M, Mazeau J: Electron impact excitation of helium: absolute differential cross sections of the $\mathbf{n}=\mathbf{2}$ and $\mathbf{3}^{3} \mathbf{S}$ states from threshold to 3-6 eV above. $J$ Phys B 1976, 9(6):933-944.

8. Brunt JNH, King GC, Read FH: A study of resonance structure in helium using metastable excitation by electron impact with high energy resolution. J Phys B 1977, 10(3):433-448.

9. Zajonc AG, Pearl JC, Zorn JC: Differential cross section for electron impact excitation of metastable helium Phys Rev A 1978, 1(4):1408-1414.

10. Hoshino M, Kato H, Tanaka H, Bray I, Fursa DV, Buckman SJ, Ingólfsson O, Brunger MJ: Benchmark differential cross sections for electron impact excitation of the $\mathbf{n}=\mathbf{2}$ states in helium at near-ionization-threshold energies. J Phys B 2009, 42:145202.

11. Fursa D, Bray I: Calculation of electron-helium scattering. Phys Rev A 1995, 52(2):1279-1297.

12. Bartschat K: Electron-impact excitation of helium from the $\mathbf{1}^{1} \mathbf{S}$ and $\mathbf{2}^{3} \mathbf{S}$ states. J Phys B: At Mol Opt Phys 1998 , 31:469-476.

13. Lange M, Matsumoto J, Lower J, Buckman S, Zatsarinny O, Bartschat K, Bray I, Fursa D: Benchmark experiment and theory for near-threshold excitation of helium by electron impact. J Phys B 2006, 39(20):4179-4190.

14. Read FH, Channing JM: Production and optical properties of an unscreened but localized magnetic field. Rev Sci Instrum 1996, 67(6):2372-2377.

15. Cubric D, Mercer DJL, Channing JM, King GC, Read FH: A study of inelastic electron scattering in He covering the complete angular range from 0 to $180^{\circ}$. J Phys $B$ 1999, 32:45-50.

16. Allan M: Excitation of the $2^{3} \mathrm{~S}$ state of helium by electron impact from threshold to $24 \mathrm{eV}$ : measurements with the 'magnetic angle changer'. J Phys B 2000, 33:L215-L220.

17. Ward R, Cubric D, Bowring N, King GC, Read FH, Fursa DV, Bray I, Zatsarinny O, Bartschat K: Differential cross sections for electron impact excitation of the $n=2$ states of helium at intermediate energies $(80,100$ and $120 \mathrm{eV}$ ) measured across the complete angular scattering range $\left(0\right.$ to $\left.\mathbf{1 8 0 ^ { \circ }}\right)$. J Phys $B$ 2011, 44(4):045209.

18. Murray AJ, Hammond P: A novel spectrometer combining laser and electron excitation and deflection of atoms and molecules. Rev Sci Instrum 1999, 70:1939-1950.

19. Feigerle CS, Pierce DT, Seiler A, Celotta RJ: Intense source of monochromatic electrons: photoemission from GaAs. Appl Phys Lett 1984, 44(9):866-868

20. Schröter CD, Rudenko A, Dorn A, Moshammer R, Ullrich J: Status of the pulsed photoelectron source for atomic and molecular collision experiments. Nucl Instrum Meth A 2005, 536(3):312-318.

21. Groves T, Hammond DL, Kuo H: Electron-beam broadening effects caused by discreteness of space charge. J Vac Sci Technol 1979, 16(6):1680-1685.

22. Kolac U, Donath M, Ertl K, Liebl H, Dose V: High-performance GaAs polarized electron source for use in inverse photoemission spectroscopy. Rev Sci Instrum 1988, 59(9):1933.

23. Blakemore JS: Semiconducting and other major properties of gallium arsenide. J Appl Phys 1982, 53(10):R123-R181.

24. Toennies JP, Winkelmann K: Theoretical studies of highly expanded free jets: influence of quantum effects and a realistic intermolecular potential. J Chem Phys 1977, 66(9):3965.

25. Haberland H, Buck U, Tolle M: Velocity distribution of supersonic nozzle beams. Rev Sci Instrum 1985 56(9):1712-1716

26. Gabriel AH, Heddle DWO: Excitation processes in helium. PRoy Soc A 1960, 258(1292):124-145

27. Moos HW, Woofworth JR: Observation of the forbidden $2^{3} S_{1} \rightarrow 1^{1} S_{0}$ spontaneous emission line from helium and measurement of the transition rate. Phys Rev Lett 1973, 30(17):775-778.

28. Van Dyck RS, Johnson CEJr, Shugart HA: Radiative lifetime of the metastable $\mathbf{2}^{1} \mathbf{S}_{\mathbf{0}}$ state of helium. Phys Rev Lett 1970, 25(20):1403-1405

29. Pretzler G: A new method for numerical Abel-inversion. Z Naturforsch 1991, 46a:639-641.

30. Killer C: Abel inversion algorithm [http://www.mathworks.com/matlabcentral/fileexchange/43639-abelinversion-algorithm]. Published 2013-09-26, Updated 2014-02-18

31. Bracewell RN: The Fourier Transform and Its Applications. Singapore: McGraw-Hill Higher Education; 2000.

doi:10.1140/epjti/s40485-014-0006-2

Cite this article as: Weyland et al:: Novel method for state selective determination of electron-impact-excitation

cross sections from $0^{\circ}$ to $180^{\circ}$. EPJ Techniques and Instrumentation 2014 1:6. 